\title{
Accidentes con Material Biológico entre Estudiantes Universitarios de Odontología
}

\author{
Daniel Antunes Freitas ${ }^{a}$, Clara Inés Vergara Hernández ${ }^{b}$, \\ Antonio Díaz Caballeroc ${ }^{c}$, Zamila Murta Morais ${ }^{d}$
}

a Profesor Titular, Facultad de Odontología FUNORTE Brasil, Campus da Saúde, Montes Claros - MG (Brasil).

${ }^{\mathrm{b}}$ Profesor Titular Tiempo Completo, Vicedecana Curricular de la Facultad de Odontología de la Universidad de Cartagena,Campus de la Salud Zaragocilla, Cartagena (Colombia).

c Profesor Titular, Facultad de Odontología, Universidad de Cartagena, Campus de la Salud Zaragocilla, Cartagena (Colombia)

d Profesor Titular, Facultad de Odontología FUNORTE Brasil, Campus da Saúde, Montes Claros - MG (Brasil).

Correspondencia: Clara Inés Vergara Hernández, Facultad de Odontología de la Universidad de Cartagena, Campus de la Salud Zaragocilla, Cartagena (Colombia). Telf.: $057+5+6698172$, Ext. 110. Correo electrónico: cvergarahdez@gmail.com.

Recibido el 1 de octubre de 2010.

Aceptado para su publicación el 28 de diciembre de 2010

\section{RESUMEN}

Objetivo. Evaluar la prevalencia de los accidentes con material biológico entre los estudiantes de pregrado en las Facultades de Odontología del Norte de Minas de las Naciones en el ejercicio de su actividad clínica.

Métodos. Estudio cuantitativo descriptivo, con una muestra de 73 estudiantes del $4^{\circ}$ al $9^{\circ}$ semestre que aceptaron participar en el proyecto. Los datos fueron tabulados y comparados por medio de análisis cuantitativo, mostrando los valores de frecuencia y porcentaje, calculados en función del número de individuos. El valor $\mathrm{p}$ fue del $5 \%$

Resultados. La edad media de los estudiantes fue de 23,6 años. En relación a los accidentes con material biológico, el $23,3 \%$ de los estudiantes expresaron una experiencia o algún tipo de accidente, y desarrollaron estos accidentes en los que utilizaban el explorador odontológico. La mayoría de los eventos ocurrió mientras realizaban la limpieza del instrumental. El órgano más frecuentemente lesionado fue la mano. La mayoría de los estudiantes que sufrieron accidentes con material biológico han participado en el programa de vacunación contra la hepatitis $\mathrm{B}$, pero no participaron en el protocolo después de la exposición, lavado de la herida con agua y jabón, y el procedimiento de guantes al momento del accidente. Mediante la prueba de Chi-cuadrado se observó una relación estadísticamente significativa entre la ocurrencia de accidentes con material biológico y el sexo $(p=0,024)$. Los varones, en un porcentaje de $83 \%$, sufren menos accidentes de riesgo biológico en comparación con las mujeres.

Conclusiones. La presencia de accidentes con instrumental entre estudiantes de odontología tiene un resultado de considerable aparición. Se deben manejar protocolos que refuercen actitudes de bioseguridad más estrictos.

Palabras clave: Estudiantes, Dental, Accidentes, Ocupacional, Sustancias Peligrosas.

\section{ABSTRACT}

Accidents with biological material among University Students of Dentistry

Objective. To evaluate the prevalence of accidents with biological material among undergraduate students in the faculties of Dentistry of Northen Minas de las Naciones.

Methods. A descriptive, quantitative study with a sample of $734^{\text {th }}$ to $9^{\text {th }}$ semester students who agreed to participate in the project. Data were tabulated and compared by means of quantitative analysis with Excel and showing frequency values and percentages calculated based on the number of individuals. The $p$ value was $5 \%$.

Results. The average student age was 23.6 years. Twenty three point three percent of the students said they experienced some kind of accident mainly when they were using a dental explorer. Most accidents occurred while cleaning instruments. The hand was the part of the body most frequently injured. Most students who had an accident had participated in the vaccination program against hepatitis B However at the time of the accident they did not participate in this program after exposure, wash the wound with soap and water or follow the procedure for gloves. The Chi-square test revealed a statistically significant relationship between the occurrence of accidents with biological material and gender $(p=0,024)$. Males, at a rate of $83 \%$, have fewer biohazard accidents compared with females.

Conclusions. There is a significant occurrence of accidents with dental material amongst dental students. Protocols should be introduced to reinforce attitudes of more stringent biosecurity. Keywords: Students, Dental, Accidents, Occupational, Hazardous Substances. 


\section{INTRODUCCIÓN}

El odontólogo y el estudiante de odontología presentan alto coeficiente de accidentabilidad debido a la práctica diaria con instrumentos rotatorios, ultrasónicos y punzocortantes, en un campo restringido de visualización y sujeto al movimiento del paciente, estando expuestos a gran variedad de microorganismos presentes en la sangre, saliva y vías aéreas respiratorias de los pacientes, así como a laceraciones accidentales con agujas y por los aerosoles contaminados con sangre y/o saliva lanzados por los equipos de alta rotación. El alto índice de accidentes ocurre en función de varios factores como: falta de atención, poco tiempo disponible, estrés, nerviosismo, exceso de fuerza y movimiento del paciente entre otros ${ }^{1-5}$.

El riesgo biológico depende de factores tales como la presencia y el volumen de sangre, la patogenicidad del agente infeccioso, las condiciones clínicas del paciente-fuente, la susceptibilidad de la persona expuesta y el adecuado seguimiento post procedimientos. La evolución de la lesión, el conocimiento sobre los agentes etiológicos, las formas de tratamientoylosfactores relacionadospermitenelestablecimiento de medidas para reducir los riesgos de salud derivados de accidentes. Se recomienda que la exposición a la sangre y otros fluidos potencialmente contaminados se considere un problema médico. Con el fin de lograr una mayor eficacia, las intervenciones para la prevención de infecciones por virus de la inmunodeficiencia humana $(\mathrm{VIH})$ y hepatitis $B(\mathrm{VHB})$ deben emprenderse inmediatamente después de la ocurrencia del accidente ${ }^{6-8}$.

La Odontología puede ser considerada como una profesión segura, siempre y cuando sean seguidos los protocolos eficaces de seguridad. Se detecta una mejoría en la conciencia y la necesidad de adoptar las medidas de precaución universal, denominadas precauciones patrón, por los profesionales de la salud a partir de la epidemia del SIDA, ya que la idea de clasificar a los individuos en grupos de riesgo en relación a la propensión de contraer y diseminar enfermedades contagiosas, realizada otrora, no tiene fundamento actual; otras patologías consideradas como potencialmente letales son la hepatitis B y C, las cuales pueden ser adquiridas por contacto a través de fluidos de los pacientes ${ }^{9-10}$.

Actualmente todos los pacientes deben ser considerados como potenciales portadores de microorganismos, pues con el uso del cóctel de medicamentos antirretrovirales es grande el número de individuos asintomáticos o que no revelan su estado de seropositividad. A pesar de esto, aún es frecuente el comportamiento individual de riesgo, como la eliminación de materiales punzocortantes en lugares inadecuados o recipientes colapsados, el transporte o manipulación de agujas desprotegidas, la desconexión de la aguja de la jeringa y principalmente el volver a tapar las agujas, que está asociado al alto potencial de riesgo de accidentes y exposición ocupacional a material biológico ${ }^{2,10-13}$.

El objetivo ha sido evaluar la prevalencia de los accidentes con material biológico entre los estudiantes de pregrado en las Facultades de Odontología del Norte de Minas de las Naciones en el ejercicio de su actividad clínica.

\section{MATERIAL Y MÉTODO}

Se trata de un estudio con enfoque cuantitativo descriptivo. Se llevó a cabo con estudiantes de colegios dentales de la Mina de Naciones del Norte en el ejercicio de la actividad clínica en el momento de la encuesta (septiembre 2009).

Se elaboró un cuestionario en dos partes: la primera con preguntas sobre la escuela de odontología, sexo y edad de los académicos, y la otra parte relacionada con la caracterización de los accidentes producidos con instrumentos cortantes, región del cuerpo afectada, circunstancia en que se produjo la exposición accidental, uso de equipos de protección individual, gravedad de la exposición, conducta adoptada después de los factores del accidente y estado actual de la vacunación contra la hepatitis B. El cuestionario fue aplicado a los estudiantes de cuarto a noveno período de FUNORTE que aceptaron participar en el estudio y firmaron un consentimiento informado.

Los datos fueron tabulados y comparados por medio de análisis cuantitativo, para lo cual fueron codificados e introducidos en formato de hoja de cálculo Excel, mostrando los valores de frecuencia y porcentaje, calculados en función del número de individuos. El valor $p$ fue del $5 \%$.

El proyecto fue aprobado por el CEP de FUNORTE 0306/99 con número de protocolo basado en estándares del Consejo Nacional de Salud/Ministerio de Salud.

\section{RESULTADOS}

De un total de 85 estudiantes, 73 de ellos, pertene- 
cientes al $4^{\circ}-9^{\circ}$ semestre estuvieron de acuerdo en participar. La edad media de los estudiantes fue de 23,6 años (DE: 4,50; rango: 19-42 años). La mayoría de los estudiantes eran mujeres y solteros (tabla 1).

En relación a los accidentes con material biológico, el $23,3 \%$ de los estudiantes declararon haber sufrido algún tipo de accidente, ocurriendo principalmente en los que utilizaban el explorador. La mayoría de los eventos ocurrió mientras se encontraban realizando la limpieza de este tipo de instrumental. El órgano más frecuentemente lesionado fue la mano, la cual presentó sangrado, alcanzando a más de la mitad de los casos (tabla 2).

Los estudiantes que sufrieron accidentes con material biológico refirieron en su mayoría la participación en el programa de vacunación contra la hepatitis B. No participaron en el protocolo después de la exposición, lavado de la herida con jabón y agua, y usar guantes de procedimiento en el momento del accidente (tabla 3 ).
Mediante la prueba Chi-cuadrado se pudo observar una relación estadísticamente significativa entre la ocurrencia de accidentes con material biológico y el sexo $(p=0,024)$. Los hombres en un $83 \%$ sufren menos accidentes de riesgo biológico en comparación con las mujeres. A su vez, las otras variables no presentaron asociación estadísticamente significativa (tabla 4).

\section{DISCUSIÓN}

La posibilidad de sufrir un accidente laboral en las áreas de las ciencias de la salud no es tan baja como se puede pensar o considerar. Los riesgos laborales o de tipo ocupacional dentro de la práctica de la odontología se incrementan cuando se trabaja bajo presión excesiva, con falta de tiempo adecuado, o con niveles variados de estrés en la población profesional odontológica, y mucho más en la población estudiantil, ya sea por la impericia, la falta de cuidados o por la falta de una exigencias de bioseguridad mucho más alta dentro de su cam-

\begin{tabular}{lcc}
\hline VARIABLES & $\mathbf{n}$ & $(\%)$ \\
\hline Edad & & \\
Hasta 22 años & 41 & $(56,2)$ \\
Mayor de 22 años & 32 & $(43,8)$ \\
Sexo & & \\
Masculino & 27 & $(37,0)$ \\
Femenino & 46 & $(63,0)$ \\
Estado civil & & \\
Soltero & 60 & $(82,2)$ \\
Casado & 10 & $(13,7)$ \\
Separado/ Divorciado & 2 & $(2,7)$ \\
Viudo & 1 & $(1,4)$ \\
Periodo que está cursando & & \\
4\% período & 7 & $(9,6)$ \\
5० & 15 & $(10,5)$ \\
$6^{\circ}$ período & 12 & $(19,4)$ \\
$7^{\circ}$ período & 14 & $(21,9)$ \\
$8^{\circ}$ período & 16 & $(12,3)$ \\
$9^{\circ}$ período & 9 & \\
\hline
\end{tabular}

Tabla 1. Caracterización de los estudiantes de odontología fuerte, septiembre 2009. 


\begin{tabular}{|c|c|c|}
\hline VARIABLES & $\mathbf{n}$ & (\%) \\
\hline \multicolumn{3}{|l|}{ Tuvo un accidente biológico. } \\
\hline Sí & 17 & $(23,3)$ \\
\hline No & 56 & $(76,7)$ \\
\hline \multicolumn{3}{|c|}{ Instrumental más usado durante el accidente. } \\
\hline Fresas & 2 & $(12,5)$ \\
\hline Explorador & 7 & $(43,6)$ \\
\hline Aguja para sutura & 2 & $(12,5)$ \\
\hline Aguja para anestesia & 4 & $(25,0)$ \\
\hline Hollemback & 1 & $(6,3)$ \\
\hline \multicolumn{3}{|c|}{ Momento del procedimiento en el que sucedió } \\
\hline Examen extraoral & 8 & $(47,1)$ \\
\hline Examen Intraoral & 9 & $(52,9)$ \\
\hline \multicolumn{3}{|l|}{ Procedimiento que estaba siendo realizado } \\
\hline Asepsia del instrumental a ser esterilizado & 2 & $(11,8)$ \\
\hline El montaje de la hoja de bisturí & 1 & $(5,9)$ \\
\hline Intercambio de fresas & 1 & $(5,9)$ \\
\hline Lavado del Instrumental & 8 & $(47,1)$ \\
\hline Preparación de la cavidad & 2 & $(11,8)$ \\
\hline Tratamiento de Endodoncia & 1 & $(5,9)$ \\
\hline Cirugía Bucal & 2 & $(11,8)$ \\
\hline \multicolumn{3}{|l|}{ Parte del organismo lesionado } \\
\hline Mano & 3 & $(17,6)$ \\
\hline Dedos & 14 & $(82,4)$ \\
\hline \multicolumn{3}{|l|}{ Presencia de sangrado } \\
\hline Si & 9 & $(52,9)$ \\
\hline No & 8 & $(47,1)$ \\
\hline
\end{tabular}

Tabla 2. Notificación de los accidentes con material biológico y las características de los accidentes en los estudiantes de Odontología Funorte, septiembre 2009.

po de formación. En el reporte actual se comprueba que la mayor accidentalidad se produce cuando se manipulan de forma poco segura instrumentos como el explorador odontológico, el cual tiene uno o dos bordes agudos que hacen mucho más fácil la presencia de eventos desagradables, como pueden ser los accidentes ocupacionales ${ }^{3,7,14}$.

La formación profesional de los futuros odontólogos debe de manera estricta incluir el conocimiento y manejo de los protocolos de bioseguridad que a nivel internacional se establecen por centros y organizaciones dedicadas a la prevención de las enfermedades transmisibles por fluidos de origen biológicos. Muchas veces la aparición de un accidente se da por el desconocimiento de unas reglas o consideraciones estipuladas, no para impedir la ocurrencia, sino para disminuir la oportunidad de que sucedan estos tipos de eventos, los cuales pueden llevar a consecuencias drásticas y de alta prevalencia de patologías, o inclusive a una mortalidad de origen ocupacional ${ }^{12,15}$.

La consideración actual que implica tratar a cada uno de los pacientes odontológicos como eventuales transmisores de patologías, como el VIH-Sida, hepatitis B y hepatitis $C$, implica la aceptación de un estricto manejo de bioseguridad para frenar la posibilidad de contagio de alguna de estas patologías mencionadas. Existe siempre una alta posibilidad en el desarrollo de las actividades clínicas y preclínicas de la profesión odontológica de adquirir una enfermedad que en ocasiones puede llegar a ser catastrófica si se obvian determinados pasos o ciertas herramientas de protección, y si a su vez se desarrollan tareas sin la atención mental y física de- 


\begin{tabular}{l|c|c}
\hline \multicolumn{1}{c|}{ VARIABLES } & $\mathbf{n}$ & $(\%)$ \\
\hline & & \\
Participó en el programa de vacunación contra la Hepatitis B & 12 & $(70,6)$ \\
$\mathrm{Si}$ & 5 & $(29,4)$ \\
$\mathrm{No}$ & & \\
$\mathrm{Se}$ adhirió a cualquier protocolo post-exposición & 7 & $(41,2)$ \\
$\mathrm{Si}$ & 10 & $(58,8)$ \\
$\mathrm{No}$ & & \\
Lavó la herida con agua y jabón & 12 & $(70,6)$ \\
$\mathrm{Si}$ & 5 & $(29,4)$ \\
$\mathrm{No}$ & & \\
$\mathrm{Registró} \mathrm{la} \mathrm{ocurrencia} \mathrm{del} \mathrm{sangrado} \mathrm{de} \mathrm{la} \mathrm{herida}$ & 5 & $(29,4)$ \\
$\mathrm{Si}$ & 11 & $(64,7)$ \\
$\mathrm{No}$ & 1 & $(6,9)$ \\
$\mathrm{Sin}$ información & & \\
Registró la profundidad de la herida & 6 & $(35,3)$ \\
$\mathrm{Si}$ & 10 & $(58,8)$ \\
$\mathrm{No}$ & 1 & $96,9)$ \\
$\mathrm{Sin}$ información & & \\
En el momento del accidente llevaba PPE & 15 & $(88,2)$ \\
$\mathrm{Si}$ & 2 & $(11,8)$ \\
No & & \\
\hline
\end{tabular}

Tabla 3. Informe de vacunación contra la hepatitis $B$ y las medidas adoptadas a raíz de un accidente con material biológico entre los estudiantes de Odontología Funorte, septiembre 2009.

\begin{tabular}{|c|c|c|c|c|c|c|}
\hline & \multicolumn{2}{|c|}{ Sufrió un accidente } & \multicolumn{2}{|c|}{ No sufrió un accidente } & \multirow{2}{*}{ OR } & \multirow{2}{*}{$\mathbf{p}$} \\
\hline & $\mathbf{n}$ & $\%$ & $\mathbf{n}$ & $\%$ & & \\
\hline \multicolumn{7}{|l|}{ Edad } \\
\hline 22 años & 10 & $(58,8)$ & 31 & $(55,4)$ & 1,00 & \\
\hline Mayor de 22 años & 7 & $(41,2)$ & 25 & $(44,6)$ & 1,15 & 0,801 \\
\hline \multicolumn{7}{|l|}{ Género } \\
\hline Masculino & 2 & $(11,8)$ & 25 & $(44,6)$ & 1,00 & \\
\hline Femenino & 15 & $(88,2)$ & 31 & $(55,4)$ & 0,17 & 0,024 \\
\hline \multicolumn{7}{|l|}{ Estado civil } \\
\hline Soltero & 14 & $(82,4)$ & 46 & $(82,1)$ & 1,00 & \\
\hline Casado/otros estados & 3 & $(17,6)$ & 10 & $(17,9)$ & 1,01 & 0,984 \\
\hline \multicolumn{7}{|l|}{ Periodo } \\
\hline $4^{\circ}$ a $6^{\circ}$ período & 5 & 29,4 & 29 & 51,8 & 1,00 & \\
\hline $7^{\circ}$ a $9^{\circ}$ período & 12 & 70,6 & 27 & 48,2 & 0,39 & 0,112 \\
\hline
\end{tabular}

Tabla 4. Factores asociados a los accidentes con material biológico entre los estudiantes de Odontología Funorte, septiembre 2009. 
bida en cada uno de esos momento de alto riesgo profesional ${ }^{9,16}$.

Al parecer, las diferencias observadas en la aparición de los accidentes y su relación con el género se pueden deber a la diferente capacidad de reportar en forma veraz y oportuna estas situaciones indeseadas o a la diferente atención y seguimiento de normas de bioseguridad ${ }^{10,14,16}$.

A la luz de las limitantes actuales, se puede concluir que los accidentes con materiales potencialmente contaminados con agentes biológicos en la Escuela de Odontología de Minas Gerais es una posibilidad real de ocurrir en momentos determinados de la práctica profesional. Al parecer, los hombres tienen menor posibilidad de sufrir accidentes en la manipulación de instrumentales cortantes y punzantes, al comparar con las mujeres. Es necesario profundizar en estos tópicos de investigación con el fin de establecer posibles asociaciones estadísticas que exijan a la población estudiantil de Odontología un enfoque de auto prevención y cuidado mucho más fuerte que lo demostrado en la actualidad.

\section{BIBLIOGRAFÍA}

1. Tillberg A, Jarvholm B, Berglund A. Risks with dental materials. Dent Mater. 2008; 24(7):940-3.

2. Machado-Carvalhais HP, Martins TC, Ramos-Jorge ML, Magela-Machado D, Paiva SM, Pordeus IA. Management of occupational bloodborne exposure in a dental teaching environment. J Dent Educ. 2007; 71(10):1348-55.

3. Oliveira AC, Lopes AC, Paiva MH. Occupational accidents due to exposure to biological material in the multidisciplinary team of the emergency service. Rev Esc Enferm USP. 2009; 43(3):677-83.

4. Petrucci C, Alvaro R, Cicolini G, Cerone MP, Lancia L. Percutaneous and mucocutaneous exposures in nursing students: an Italian observational study. J Nurs Scholarsh. 2009; 41(4):337-43.
5. Li Y, Mei GY, Jiang HY, Wang GR, Wei Q, Han L, et al. Study on the phenomenon of splashes and sprays from virology. Zhonghua Shi Yan He Lin Chuang Bing Du Xue Za Zhi. 2009; 23(6):458-60.

6. Vieira M, Padilha MI. HIV and the nursing professional in the face of needlestick accidents. Rev Esc Enferm USP. 2008; 42(4):804-10.

7. Gir E, Netto JC, Malaguti SE, Canini SR, Hayashida M, Machado AA. Accidents with biological material and immunization against hepatitis $B$ among students from the health area. Rev Lat Am Enfermagem. 2008; 16(3):401-6.

8. Gutierrez EB, Lopes MH, Yasuda MA. Accidental exposure to biological material in healthcare workers at a university hospital: Evaluation and follow-up of 404 cases. Scand J Infect Dis. 2005; 37(4):295-300.

9. Garcia de Codes Ilario A, de Juanes Pardo JR, Arrazola Martinez Mdel P, Jaen Herreros F, Sanz Gallardo MI, Lago Lopez E. Accidents with exposure to biological material contaminated with HIV in workers at a third level hospital in Madrid. Rev Esp Salud Publica. 2004; 78(1):41-51.

10. Reis RK, Gir E, Canini SR. Accidents with biological material among undergraduate nursing students in a public Brazilian university. Braz J Infect Dis. 2004; 8(1):18-24.

11. Murofuse NT, Marziale MH, Gemelli LM. Accidents with biological material at West Parana University Hospital. Rev Gaucha Enferm. 2005; 26(2):168-79.

12. Manetti ML, da Costa JC, Marziale MH, Trovo ME. Prevention of occupational accidents with biological material as per Green and Kreuter Model. Rev Gaucha Enferm. 2006; 27(1):80-91.

13. Machado-Carvalhais HP, Ramos-Jorge ML, Auad SM, Martins LH, Paiva SM, Pordeus IA. Occupational exposure to potentially infectious biological material in a dental teaching environment. J Dent Educ. 2008; 72(10):1201-8.

14. Chiodi MB, Marziale MH, Robazzi ML. Occupational accidents involving biological material among public health workers. Rev Lat Am Enfermagem. 2007; 15(4):632-8.

15. Canini SR, Gir E, Machado AA. Accidents with potentially hazardous biological material among workers in hospital supporting services. Rev Lat Am Enfermagem. 2005; 13(4):496-500.

16. Hernandez Navarrete MJ, Montes Villameriel FJ, Solano Bernad VM, Sanchez Matienzo D, del Val Garcia JL, Gil Montalban E, et al. Accidents with biological material in health care workers in 2 primary health care areas (19901999). Aten Primaria. 2001; 28(4):255-8. 